\title{
A Rare Case of Leiomyosarcoma of Inferior Vena Cava Managed with Curative Resection and PTFE Vascular Graft Reconstruction: A case report with review of literature
}

\author{
Gori $\mathbf{J}^{1}$, Nithya $\mathbf{M}^{2}$, Bhaduri $\mathrm{D}^{3}$, Bhatia $\mathbf{M}^{4}$ \\ ${ }^{1}$ Dr Jayesh Gori, Registrar, Department of Surgical Oncology, ${ }^{2}$ Dr Nithya Manyath, Senior Resident, Department of Surgical \\ Oncology, ${ }^{3}$ Dr Debanshu Bhaduri, Primary Consultant Oncosurgeon, Department of Surgical Oncology, ${ }^{4}$ Dr Manish Bhatia, \\ Primary Consultant Oncosurgeon, Department of Surgical Oncology. All are affiliated with M.N.B.C.I., Inlaks and Budharani \\ Hospital, Pune, Maharashtra, India
}

Corresponding Author: Dr Jayesh Gori, E-mail: jayeshgori26feb@gmail.com

\begin{abstract}
Primary vascular leiomyosarcoma is rare tumour arising most frequently from inferior vena cava. In literature only case reports or small case series are available. The presentation and surgical management depends on origin of the tumour in relation to the hepatic and renal veins, tumour growth (extra luminal or intra luminal) and involvement of surrounding structures. Leiomyosarcoma of inferior vena cava tumour poses a challenge with respect to resectability, reconstruction, potential for massive haemorrhage, requirement of cardiopulmonary bypass and possibility of tumour thromboemboli. Our experience with successful management of rare case and with thorough literature review suggest surgical resection of leiomyosarcoma of the IVC with Polytetrafluoroethylene vascular graft reconstruction provides good surgical and oncological outcome.
\end{abstract}

Key-words: Leiomyosarcoma, Inferior Vena cava, Polytetrafluoroethylene Vascular Graft, Cardiopulmonary Bypass.

\section{Introduction}

Leiomyosarcoma of inferior vena cava (IVC) accounts for only $0.5 \%$ of all soft tissue sarcomas in adults and it is a rare tumor of mesenchymal origin and arises from the smooth muscle cells of the tunica media, predominantly within the wall of inferior vena cava. In literature less than 300 cases being reported [1]. Extra luminal or intraluminal or both tumor growth can be seen but extra luminal seems to be the common presentation $[1,2]$. They are more common in females and mostly seen in the sixth decade [3]. Symptoms and management varies according to the dimension, growth pattern, and localization of the tumour. The only therapeutic option associated with prolonged survival are radical en bloc resection of the affected venous segment $[1,4]$. We are presenting the case of 43 year female patient with level 2 Leiomyosarcoma of IVC managed with curative resection with polytetrafluoroethylene vascular graft reconstruction.

\section{Case History}

A 43 year female patient presented with upper abdominal pain with discomfort since 3 months with minimal tenderness in epigastric region. The $\mathrm{CT}$ abdomen was

International Journal of Medical Research and Review suggestive of well circumscribed smooth margined retroperitoneal soft tissue density $(34-56 \mathrm{HU})$ mass of $36 \times 26 \times 43 \mathrm{~mm}$ involving anterior wall of the IVC. The mass was extending from the lowermost intrahepatic segment of IVC up to the confluence of the renal veins and protruding into the lumen nearly completely occluding it. The anterior extra luminal component was indenting the head of pancreas, portal vein confluence, $\mathrm{CBD}$, caudate lobe of liver and second part of duodenum with well-preserved planes (Figure1).

After confirming no metastasis in lung and liver with radiological imaging, we planned surgery in cardiac operation theatre with cardio thoracic surgeons. Right thoraco- abdominal incision was taken and small and large bowel mobilized. A5x6 cm sized circumscribed tumour arising from the anterior wall of IVC, extending onto the lateral wall with preserved planes with surrounding structure was identified. The upper edge of the tumour was extending just short of retro hepatic IVC (Figure 2A). Liver was mobilized by dividing its attachment with the diaphragm. Both renal veins were identified and looped as was the right renal artery. Available online at: www.ijmrr.in 249 | P a g e 


\section{Case Report}

Vascular control was achieved by applying clamps over both renal veins, IVC on either side of the tumour and lumbar veins draining in the intervening segment of IVC but she remain hemodynamically stable (Figure 2B). Tumour along with IVC wall excised and sent for frozen section to confirm negative margin excision. The IVC

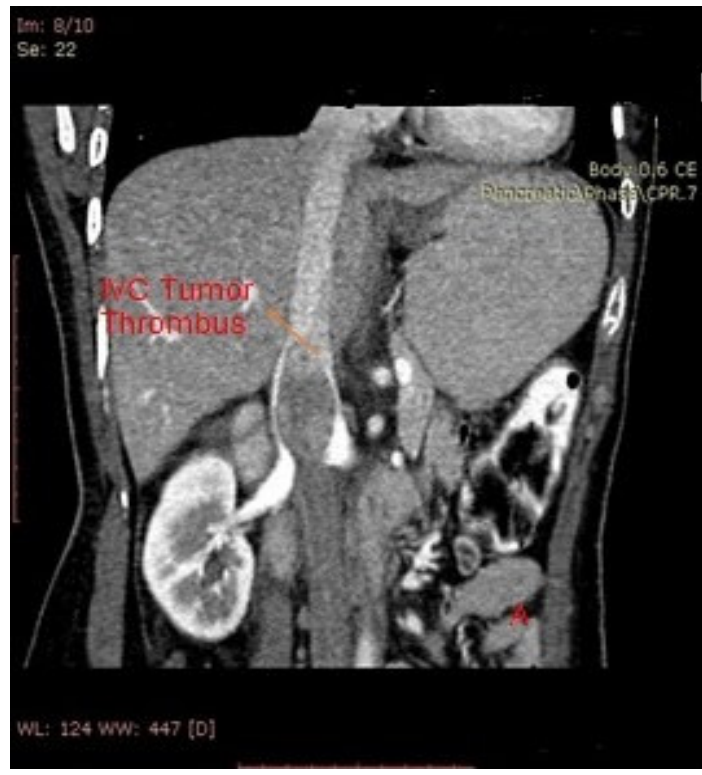

defect was closed with $16 \mathrm{~mm}$ partial regular wall PTFE graft sutured with 6-0 Prolene (Figure 3A). After appropriate flushing manoeuvres to check adequate flow and haemostasis, vascular clamps were removed. Patient tolerated procedure well. Post-operative period remain uneventful.

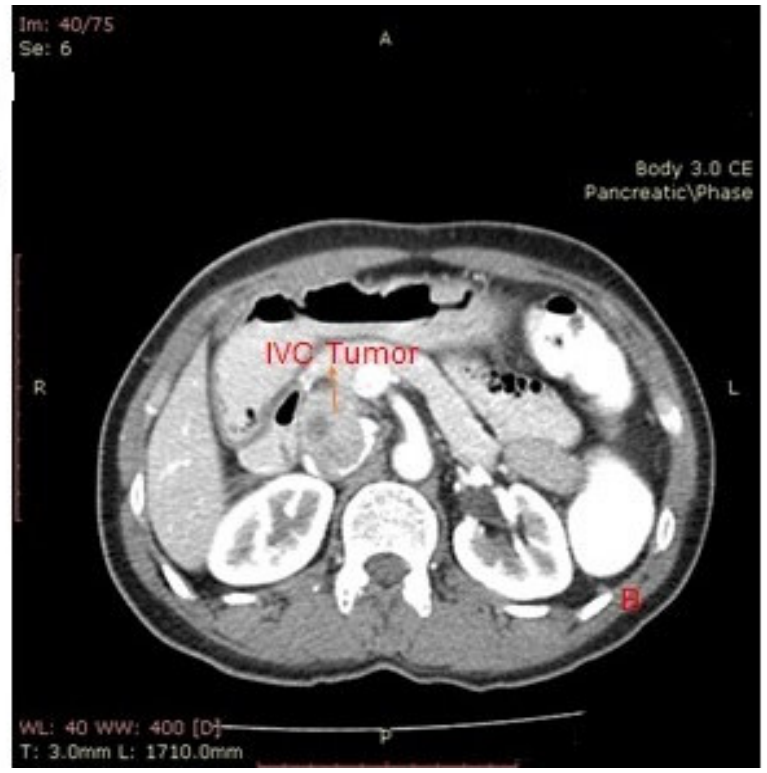

Figure 1: C.T. scan showing (A) well circumscribed retroperitoneal soft tissue mass extending from the lowermost intrahepatic segment of IVC up to the confluence of the renal veins, protruding into the lumen. (B) The anterior extra luminal component indenting the surrounding structure.
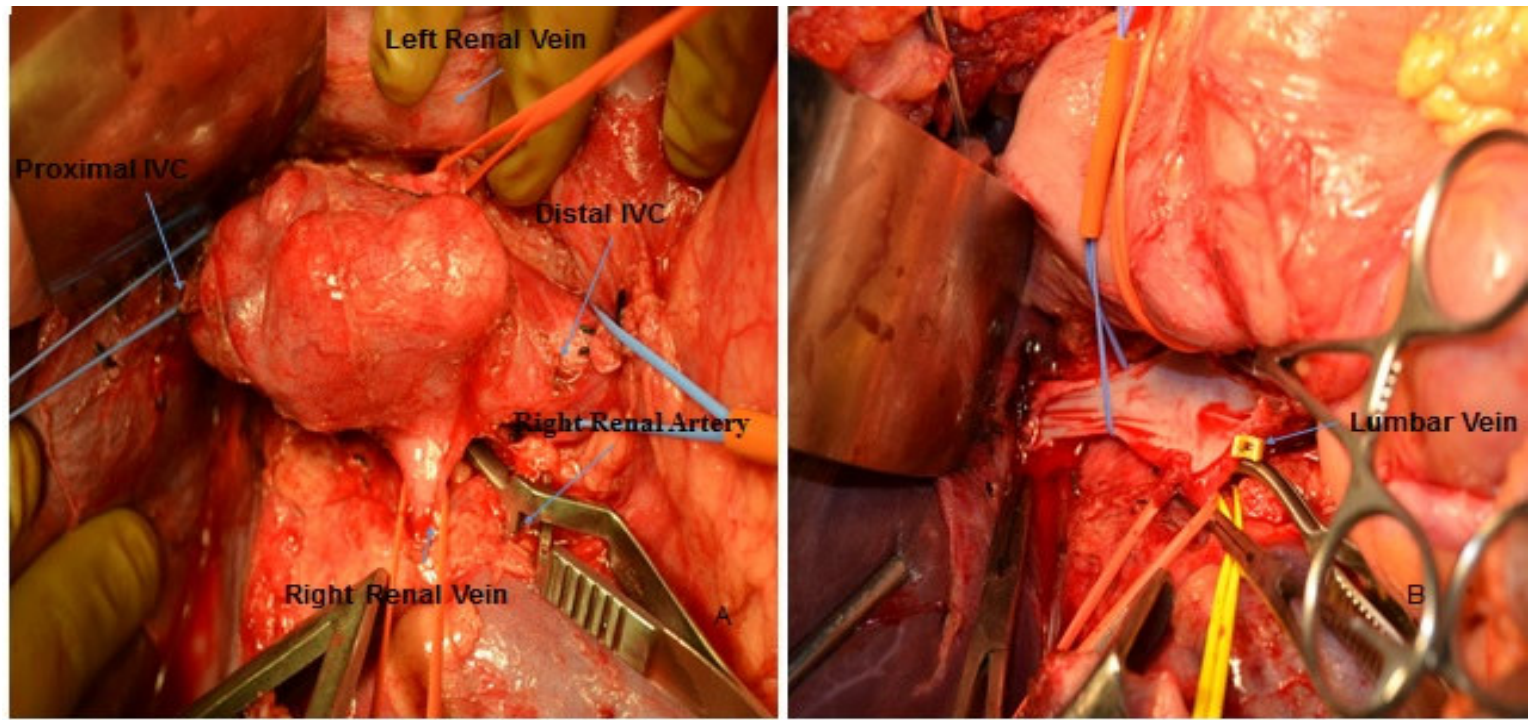

Figure 2: Intra-operative Picture showing (A) Proximal and distal control of IVC, along with control of the renal and lumbar veins. (B) Tumour along with wall of IVC excised

On sectioning the IVC, the cut surface showed a $5.5 \times 3 \times 2.5 \mathrm{~cm}$, well encapsulated, firm and pink-tan to white lobulated mass with whorled bulging cut surface (Figure 3B). The microscopic examination was suggestive of spindle cell tumour arising from smooth muscle, with focal increase in mitosis. The malignant spindle cells had elongated eosinophilic cytoplasm and 


\section{Case Report}

elongated nuclei with rounded ends,the cells were arranged in fascicles. Haemorrhage and necrosis was seen consistent with leiomyosarcoma with all margins free (Figure 4).Patient was kept on oral anticoagulant for 6 months and then stopped. She is at present 15 months of regular follow up with USG Abdomen with colour doppler and chest x-ray 3 monthly and yearly CT scan abdomen is asymptomatic with no signs of recurrence.
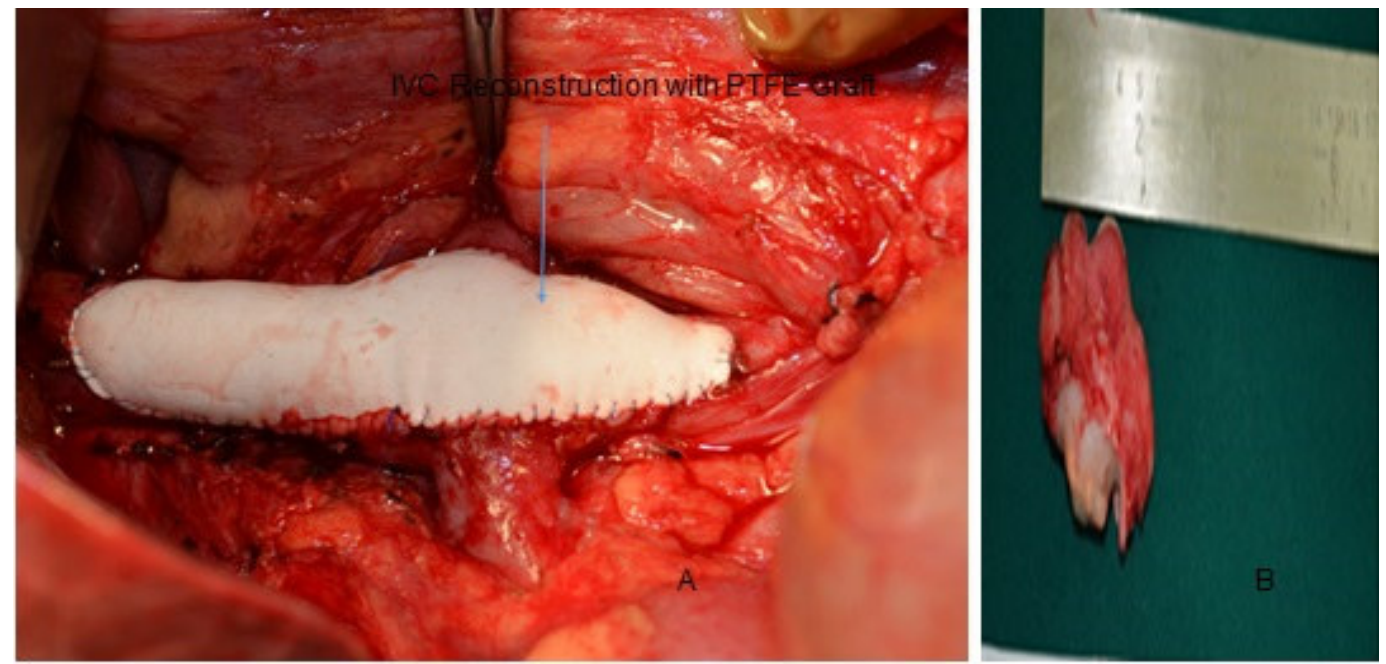

Figure 3: (A) IVC reconstruction with PTFE vascular graft. (B) Well encapsulated pink-tan to white lobulated mass
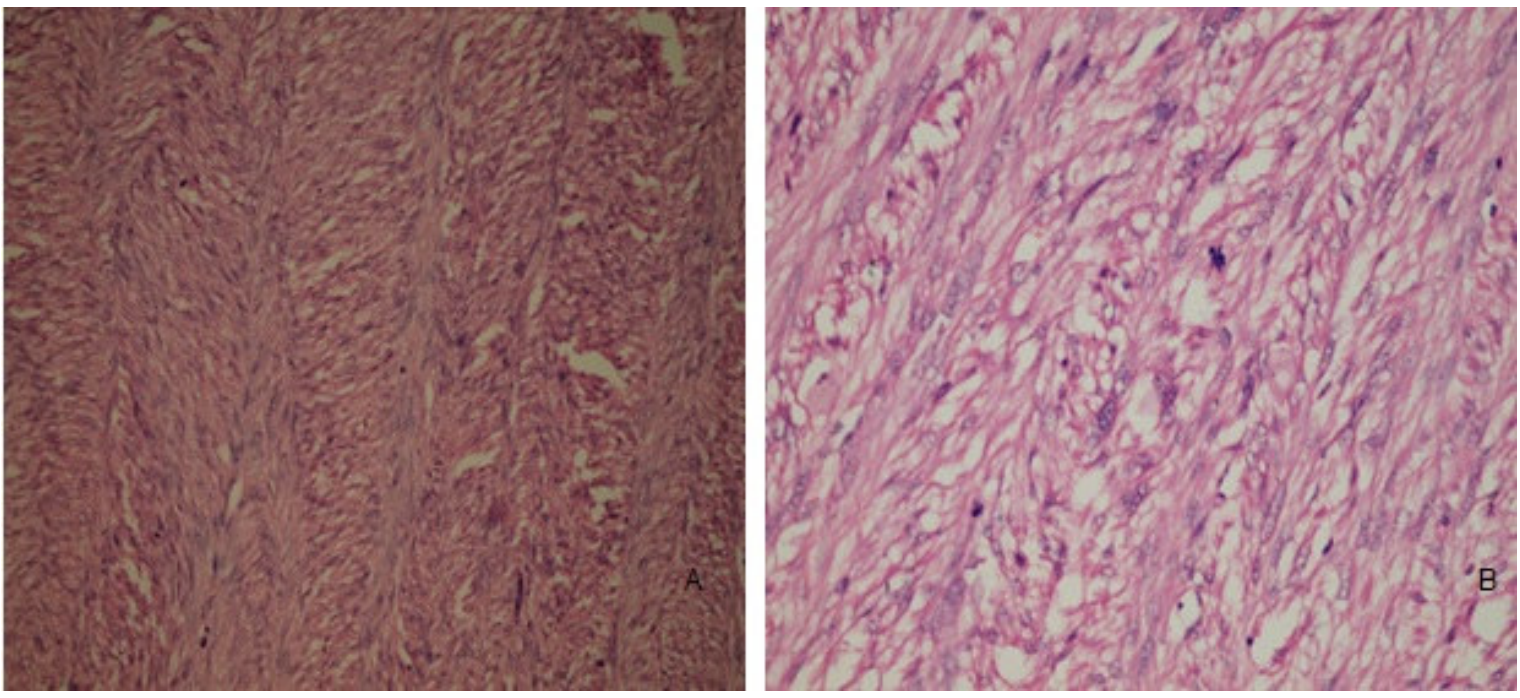

Figure 4: Microphotograph of $\mathrm{H}$ and $\mathrm{E}$ stain showing (A) At 200X magnification many spindle cells arranged in fascicles with haemorrhage and few necrosis seen (B) At 400X spindle cells with focal increase in mitosis, elongated nuclei with rounded ends and eosinophilic cytoplasm.

\section{Discussion}

Leiomyosarcoma of the IVC are extremely rare, documented in the surgical literature mostly as case reports with few small case series.Curative treatment of choice being complete surgical resection with a tumourfree margin of $1 \mathrm{~cm}$ [3].Pre-operative tissue diagnosis is not mandatory for surgery. Curative options available are ligation, resection and cavoplasty with or without graft replacement. Operative management are challenging for tumours involving middle level (segment II) and especially for upper level(segment III) [4]. In our case complete resection of tumour with $\mathrm{R} 0$ margin was possible because of right thoracoabdominal approach with liver mobilization that gives good exposure of IVC with adequate proximal and distal control of caval flow. 
Prosthetic replacement is favored because it allows a more radical approach and replacement for circumferential resected segment of vein. This technique is preferred in patients with large intramural and intraluminal tumors growth. Theoretically this procedure carries an increased risk of pulmonary embolism as well as further graft-related major complications such as sepsis, graft occlusion and graft-enteric fistulas. Whether long term anticoagulation is truly required or not is still controversy since it also carries the potential of hemorrhage. Several cases of postoperative graft occlusions were reported [5]. Thus, we preferred to continue anticoagulation for the 6 months. Ringed reinforced PTFE graft is prosthesis of choice for IVC replacement because it provides the best results, given the length of the missing segment and the need for strength to resist compression in the abdomen. In literature 14 to 20 mm diameter PTFE graft mentioned but many authors prefer a 20-mm-diameter graft for best congruency with the native vessel and some recommend smaller grafts (14 - $16 \mathrm{~mm}$ ) for infrarenal replacement to increase blood velocity [6]. In our case due to large tumor with more than $50 \%$ circumference involvement simple cavoplasty was not possible. We had used $16 \mathrm{~mm}$ partial circumference PTFE vascular graft. Even after radical surgery prognosis remain poor and over half of patients will develop tumor recurrence. The 5-year survival rate ranges between $31 \%$ and $62 \%$ [7]. In our case till total of 15 month follow up there was no recurrence, graft-enteric fistula or thrombosis.

Hines et al. reported improved survival with combined postoperative chemo radiation. However, the benefit of radiation, chemotherapy, or both for the treatment of IVC leiomyosarcoma is currently uncertain [6]. We had not given adjuvant treatment due to able to get adequate margin and potential radiation associated complications like graft enteric fistula.

\section{Conclusion}

Our experience with successful management of a rare case and with thorough literature review suggests curative surgical resection of leiomyosarcoma of the IVC with PTFE graft reconstruction provides good surgical and oncological outcome. The role of adjuvant treatment still remains uncertain.

\section{Conflicting Interest:None To Declare}

Funding: Nil

\section{References}

1. Alexander A, Rehders A, Raffel A, Poremba C, Knoefel WT, Eisenberger CF. Leiomyosarcoma of the inferior vena cava: radical surgery and vascular reconstruction. World J Surg Oncol. 2009 Jan 26;7:56.

2. Hartman DS, Hayes WS, Choyke PL, Tibbetts GP. From the archives of the AFIP. Leiomyosarcoma of the retroperitoneum and inferior vena cava: radiologicpathologic correlation. Radiographics. 1992 Nov 12(6):1203-20.

3. Hemant D, Krantikumar R, Amita J, Chawla A, Ranjeet N. Primary leiomyosarcoma of inferior vena cava, a rare entity: Imaging features. Australas Radiol. 2001 Nov 45(4):448-51.

4. Kieffer E, Alaoui M, Piette J-C, Cacoub P, Chiche L. Leiomyosarcoma of the inferior vena cava: experience in 22 cases. Ann Surg. 2006 Aug 244(2):289-95.

5.Bower TC, Nagorney DM, Cherry KJ, Toomey BJ, Hallett JW, Panneton JM, et al. Replacement of the inferior vena cava for malignancy: an update. J Vasc Surg. 2000 Feb 31(2):270-81.

6. Hines OJ, Nelson S, Quinones-Baldrich WJ, Eilber FR. Leiomyosarcoma of the inferior vena cava: prognosis and comparison with leiomyosarcoma of other anatomic sites. Cancer. 1999 Mar 185(5):1077-83.

7. Ito H, Hornick JL, Bertagnolli MM, George S, Morgan JA, Baldini EH, et al. Leiomyosarcoma of the inferior vena cava: survival after aggressive management. Ann Surg Oncol. 2007 Dec 14(12):3534-41.

\section{How to cite this article?}

Gori J, Nithya M, Bhaduri D, Bhatia M. A Rare Case of Leiomyosarcoma of Inferior Vena Cava Managed with Curative Resection and PTFE Vascular Graft Reconstruction: A case report with review of literature. Int J Med Res Rev 2014;2(3):249-252. doi:10.17511/ijmrr. 2014.i03.13. 